\section{Entgrenzte Körper \\ Zur Darstellung von Körperausscheidungen in der Neuen Deutschen Popliteratur}

Mitten in der Nacht wache ich auf, und es riecht so komisch im Zimmer, und ich schlage die Augen auf und fühle im Dunkeln so um mich herum, und alles ist naß und ich denke: Um Gottes willen. Feuchten Traum gehabt. Bitte, bitte, bitte nicht jetzt und nicht hier. Ich mache also das Licht am Nachttisch an, Knips macht das, und ich gucke an mir herunter und sehe, daß ich ins Bett gekotzt habe, aber das ist nicht alles, nein, ich habe auch noch ins Bett geschissen. ${ }^{1}$

Als im Jahre 1995 der Debütroman Christian Krachts Faserland erscheint, schreibt Ilka Piepgras in der »Berliner Zeitung«:

Barbour-Jacken werden angezogen, ausgezogen, vergessen, verbrannt, geklaut. Ansonsten wird viel gesoffen und noch mehr gekotzt. Eigentlich wird ständig gekotzt und zudem manchmal ins Bett geschissen. Viel mehr tut sich nicht. Weil aber drastische Sprache und drastische Episoden nicht über Substanzlosigkeit hinwegtäuschen können, bleibt nach der Lektüre des Romans nur eine große Leere. $^{2}$

Piepgras, ebenso wie zahlreichen anderen Rezensenten, dienten die offensive Darstellung von Körperausscheidungen und das scheinbare Fehlen eines Handlungsstranges als Merkmale
Das Motiv der

Körperflüssigkeiten wird als ein Grundmotiv der Neuen Deutschen Popliteratur vorgestellt. Die in Verbindung mit diesem Motiv inszenierten Körperbilder können als entgrenzt beschrieben werden. Das Konzept der Entgrenzung fußt auf unterschiedlichen, kulturhistorisch etablierten Zuschreibungen und Kontextualisierungen, die erläutert und im Anschluss als Basis der exemplarischen Textanalyse dienen. Unter besonderer Fokussierung der vorgeführten Fäkalien werden die Romane Feuchtgebiete (2008) von Charlotte Roche und Fleckenteufel (2009) von Heinz Strunk untersucht. 
literarischer Minderwertigkeit. ${ }^{3}$ Der Roman, der trotz seiner zum Teil kontroversen Kritiken zum Bestseller avancierte und heute zum festen Bestandteil germanistischer Lehre zählt, fand zahlreiche Nachahmer, die sich ähnlicher Verfahren und Themen bedienten. Benjamin von Stuckrad-Barre schildert in Livealbum (1999) detailliert die durch den ständigen medialen Anpassungsdruck ausgelöste Bulimie seiner Erzählfigur:

Noch tiefer ins Klo beugen, Finger jetzt in den Hals, es passiert nichts. Richtig hinten rein, vielleicht zwei Finger. Waren das die Mandeln? Das tat weh, das schien der richtige Weg. Krampf. Oh ja, es zuckte. Es ging, es kam, das mußten sie sein, die Mohrenköpfe. Stellung halten, mit der rechten Hand nach oben, tasten nach dem Knopf, spülen und weiter. In die Fluten Kotzen, es jetzt von selbst. [...] Die Seele aus dem Leib, sagt man ja so. ${ }^{4}$

In Tristesse Royale (1999) des popkulturellen Quintetts wird das unvermittelte und/oder bewusst herbeigeführte Sichentäußern als Zivilisationskrankheit im Kontext des Überflusses stilisiert, und in Relax (1997) von Alexa Henning von Lange dienen die Sekrete des Körpers vorrangig zur Verschleierung einer längst gescheiterten Paarbeziehung. ${ }^{5}$ Christian Kracht selbst setzt die Körpersäfte Blut, Schweiß, Fäkalien und Urin in 1979 (2001) als Symbol für den Zwang zur Entindividualisierung des Ich-Erzählers ein. All diese Texte, die unmittelbar mit dem Pop-Etikett versehen wurden, thematisieren Musik und Marken ebenso wie Konsum und Kotze. Die Figuren der Popliteratur haben, so urteilte bereits 2001 Georg Seeßlen, in der Mehrzahl eine Manie für beides, »für die glänzende Oberfläche der Warenwelt und aber auch für die unkontrollierte, konvulsivische [...] Offenheit des Körpers «. ${ }^{6}$ Rückblickend kann festgehalten werden, dass die Konsumgüter ebenso zu einem Grundmotiv der Popliteratur avancierten wie die Sekrete des Körpers. ${ }^{7}$ Frank Degler liest in der Verbindung dieser beiden Motive eine »epochale Pathologie «, ${ }^{8}$ die er als »Mangel eines Mangels $~^{9}$ beschreibt. Er resümiert:

Die Epochenkonstellation, auf die die Körper der Ich-ErzählerInnen reagieren, ist die eines ubiquitären Überflusses an Konsummöglichkeiten, an Kommunikationsangeboten, Nahrungs- und Genussmitteln, Glücksversprechen, sexuellen Lüsten und Freiheiten. Die

3 Zum Verhältnis von literarischer Wertung und der Darstellung von Körpersekreten vgl. Meinen: Eine Ästhetik des Ekels, S. 113-124.

4 Stuckrad-Barre: Livealbum, S. 104f.

5 Eine quantitative Aufstellung der Sekretdarstellungen in den Texten Faserland (Christian Kracht), Relax (Alexa Henning von Lange), Soloalbum (Benjamin von Stuckrad-Barre), Livealbum (Benjamin von Stuckrad-Barre), Tristesse Royale (Joachim Bessing, Christian Kracht u.a.) und 1979 (Christian Kracht) findet sich bei Degler: Sekrete Kommunikation, S. 265-287.

6 Seeßlen: Bedeutung und Wixis.

7 Vgl. Degler: Sekrete Kommunikation, S. 265-287.

8 Ebd., S. 283.

9 Ebd., S. 284. 
Körper reagieren auf dieses libidinöse Aufsaugen, indem sie dem Dauerzustand der Akkumulation und Konsumtion, Momente der Eruption und des Ausstoßes entgegensetzen. ${ }^{10}$

Degler deutet das Motiv der Körperflüssigkeiten folglich vorrangig als Ausdruck einer Zivilisationskritik im Kontext des Übermaßes. Im Gegensatz dazu kann die literarische Darstellung des sich öffnenden, unverschlossenen Körpers jedoch auch als ästhetische Neuerung verstanden werden. Im deutschsprachigen Literaturdiskurs gaben insbesondere die Popliteratinnen und Popliteraten den Startschuss für eine literarische Ästhetik, die seit einer Ausstellung von 1993 im Whitney Museum of American Art in New York unter dem Stichwort $>$ abject art ${ }^{11}$ verhandelt wird. Diese im deutschsprachigen Raum als `Ekelkunst` titulierte Ästhetik präsentiert im Motiv der Körpersekrete ein Bild des Körpers, das kontrastiv zum idealschönen verschlossenen Körperbild der Warenwelt steht, denn der popmoderne Freizeitkörper wird im Motiv der Körpersekrete als geöffnet inszeniert. Diese in der Gegenwartsliteratur präsentierten Körper weisen versehrte

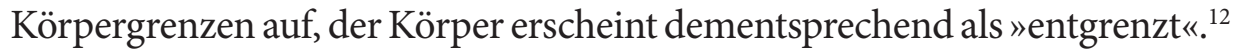
Helga Gallas spricht angesichts dieser zu beobachtenden Tendenz in der Literatur der 1990er Jahre sogar von einem »Versinken im Entgrenzten «. ${ }^{13}$

\section{Der entgrenzte Körper}

Mit der Verwendung der Begriffe `versehrt` und `entgrenzt « in Bezug auf den Körper (den - wie oben gezeigt - auch die zeitgenössische Literaturwissenschaft für die analytische Beschreibung einsetzt) geht eine Konzeptualisierung einher, derzufolge es eine Grenze zwischen einem >Innen< und einem >Außen « des Körpers gibt. Diese Grenze wird im subjektiven Erleben durch die Haut gebildet. ${ }^{14}$ Die Grenze ist weder unüberschreitbar noch undurchlässig: zum Einen durch die Existenz verschiedenster Körperöffnungen, durch die Stoffe aus dem Inneren hinaus als auch hinein können, zum Anderen durch die Tatsache, dass die Haut eine durchlässige Membran ist, die z.B. Schweiß absondert als auch Stoffe aus der Umgebung aufnehmen kann. Obwohl diese Austauschprozesse unausweichlich sind,

13 Gallass: Kleists Welt im Vergleich zur Inflation des Realen in der Gegenwartsliteratur, zit. nach Rytz: Die Sprache der Haut, S. 44.

14 Zur Grenzmetapher vgl. Benthien: Haut, S. 25-48. 
hat sich ab dem 18. Jahrhundert im Zusammenspiel des medizinischen ${ }^{15}$ und ästhetischen ${ }^{16}$ Diskurses, in dem der verschlossene - und damit ’hygienische $<$ - Körper als Ideal gesetzt. ${ }^{17}$ In diesem Diskurs wird eine rigide Eindämmung und zugleich Regulierung der Zirkulation von Körpersekreten propagiert. Michail Bachtin hat diesen Wandel ausführlich beschrieben, im neuen Körperkanon wird »der fertige, streng begrenzte, nach außen verschlossene, von außen gezeigte, unvermischte und individuelle und ausdrucksvolle Körper«idealisiert.

Alles, was absteht und vom Körper ausgeschieden wird, alle deutlichen Ausbuchtungen, Auswüchse und Verzweigungen, d.h. all das, womit der Körper über seine Grenze hinausgeht und wo ein anderer Körper anfängt, wird abgetrennt, beseitigt, verdeckt und gemildert. Ebenso werden alle ins Körperinnere führenden Öffnungen geschlossen. ${ }^{18}$

Die Körpergrenzen dieser idealschönen Leiber sind jedoch - wie Menninghaus herausarbeitet - einer permanenten Bedrohung ausgesetzt, denn der »undurchlässige, geschlossene, makellose Behälter-Körper gerät in die Position der reinen und idealen Ordnung, während alle Öffnungen, Randbereiche und Oberflächen-Defekte die Gefährdung durch Verunreinigung markieren. ${ }^{19}{ }^{19}$ Die historische Entwicklung des Körperdiskurses zeigt also deutlich, warum Sekrete des Körpers im Rahmen der Literatur seit der zweiten Hälfte des 18. Jahrhunderts vorrangig im Kontext des Ekels, des Disparaten, des Pathologischen, des Grotesken und/oder des Hässlichen konstruiert wurden - und warum die offensive, nicht negativ wertende Darstellung des geöffneten, nicht klar `begrenzten Körpers als ästhetische Kehrtwende in der Gegenwartsliteratur verstanden werden kann, wie die folgenden Ausführungen zeigen werden.

Dem Konzept der (Körper-)Grenze ist immer auch die Möglichkeit des Grenzübertritts immanent. Körperausscheidungen können in diesem Sinne als `Grenzübertritte « verstanden werden. Zwar sind sie biologisch unausweichlich, unterliegen jedoch im gewissen Maße der Kontrolle des Individuums. Dies ist die Voraussetzung dafür, dass sie Gegenstand von kulturspezifisch und historisch wandelbaren Regeln und Konventionen werden, innerhalb derer sich ein Regelsystem etabliert, das Auskunft darüber gibt, wer, wann, wie und wo die Grenze überschreiten kann und

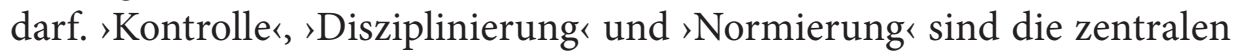
Stichworte innerhalb eines Körperdiskurses, der nach der Funktion des

Vgl. Löw: Der Körperraum als soziale Konstruktion, S. 211-222.

16 Vgl. Meinen: Eine Ästhetik des Ekels, S. 113-124.

17 Zur symbolischen Aufladung der Körperöffnungen vgl. Douglas: Reinheit und Gefährdung.

18 Bachtin: Rabelais und seine Welt, S. 361.

19 Menninghaus: Ekel, S. 154. 
menschlichen Körpers innerhalb zivilisierter Gesellschaften fragt. In seinem Werk Über den Prozess der Zivilisation formuliert Norbert Elias mit Blick auf den Zivilisationsprozess: »[...] das Verhalten von immer mehr Menschen muß aufeinander abgestimmt, das Gewebe der Aktionen immer genauer und straffer durchorganisiert sein, damit die einzelne Handlung darin ihre gesellschaftliche Funktion erfüllt. $\aleph^{20}$ Hiervon sei vorrangig der menschliche Affekthaushalt betroffen, der einer Funktionalisierung des Einzelnen im Rahmen dieses Prozesses entgegenstünde. Systematische Affektkontrolle sei demnach Grundbedingung in beständig expandierenden und sich demzufolge ausdifferenzierenden Gesellschaften. Die Affektkontrolle sei vorrangig dem Einzelnen übertragen worden, wie Elias am Verschwinden der Gewalt aus dem öffentlichen Raum erläutert. Das Unsichtbarwerden der Gewalt durch deren Monopolisierung ließe einen "Selbstkontrollapparat « entstehen, der die Einzelnen an ein "geregeltes Ansich-Halten « gewöhne. ${ }^{21}$ Dieses »Ansich-Halten «, so ließe sich daran anschließend formulieren, wird in der `zivilisierten ‘ Gesellschaft nicht nur für die Affekte, sondern auch für die biologisch begründeten Ausscheidungsprozesse des Körpers gefordert.

Auf diesem Verständnis aufbauend, thematisieren die Texte der Popliteratur im Rahmen der Darstellung von Körpersekreten zwei Aspekte: Einerseits zeichnen sie im Motiv der Körpersäfte ein Bild von der Unmöglichkeit der vollständigen Selbstkontrolle, andererseits wird in den Sekreten die Frage nach einem bewussten Widerstand gegen Disziplinierung und Normierung innerhalb eines Körperdiskurses aufgeworfen, der eine vollständige Körperverschließung fordert.

Für eine derartige Auseinandersetzung gaben die Texte der 1990er Jahre Anstoß. Während die Arbeiten Krachts, Stuckrad-Barres und von Langes jedoch letztlich im pathologischen Diskurs verbleiben, entstehen ab den 2000er Jahren Texte, die, an popliterarische Ausdrucksformen anschließend, den pathologischen Diskurs zunehmend in den Hintergrund treten lassen und den Körper und seine Ausscheidungen auch als lustvolle Entgrenzungen zu inszenieren suchen. Hierzu werden im Folgenden zwei Texte detailliert besprochen, die in Inhalt und Form, trotz deren vermeintlichem Ende, zur Popliteratur zu zählen sind: Feuchtgebiete (2008) der ehemaligen VIVAModeratorin Charlotte Roche und Fleckenteufel (2009) von Heinz Strunk. Die Dichte und Intensität, mit der die Körpersekrete und -öffnungen in diesen Texten inszeniert werden, ist in diesen Werken besonders auffällig. Der Debütroman Charlotte Roches ist nach Aussage der Autorin »ausschließlich 
dem Bereich zwischen Muschi und Poloch gewidmet « ${ }^{22}$ und Heinz Strunk erhebt den Stuhlgang seines pubertierenden Protagonisten in Fleckenteufel zum Leitmotiv seines Romans. Sowohl Roche als auch Strunk sind Teil des Kultur- und Medienbetriebes. Bei ihren Erzählungen handelt es sich um Konstruktionen eines generationsspezifischen Archivs, dessen jeweiliger Gegenwartsbezug vorrangig durch popkulturelle Referenzen hergestellt wird. Inhaltlich sind die Erzählungen auf das Beobachtbare, das alltägliche Detail reduziert und durch die konsequente Subjektivität der jeweiligen Erzählinstanzen geprägt. ${ }^{23}$ Deutlicher noch als in den Texten von StuckradBarre, Kracht und von Lange wird in diesen beiden Erzählungen nach den Möglichkeiten einer Lebensweise gefragt, die sich nicht uneingeschränkt dem Diktat der Verschließung unterwirft und zugleich die Konstruktivität der kultur- und zeitbedingten Grenzübertrittsmodalitäten verhandelt.

\section{Charlotte Roche: Feuchtgebiete}

Der Debütroman von Charlotte Roche zeichnet ein differenziertes Körperbild, das im direkten Kontrast zur glatten und undurchlässigen Oberfläche eines perfektionierten weiblichen Körpers steht. Roche erzählt die Geschichte der 18-jährigen Helen Memel, die nach einer missglückten Intimrasur mit einer Analfissur in der proktologischen Abteilung des Maria-Hilf-Krankenhauses liegt. Während Helen auf den Besuch ihrer geschiedenen Eltern wartet, in der Hoffnung, diese an ihrem Krankenbett wieder versöhnen zu können, erfährt der Leser gleichermaßen detailliert etwas über Helens Familie wie über ihre sexuellen Vorlieben, über Hygiene und ihre Körperöffnungen. Es ist die Rede von eiternden Pickeln, Smegma und Blut ebenso wie von Urin, Sperma und Fäkalien. Die Intensität, Frequenz und Differenziertheit, mit der Roche den geöffneten Körper als Movens der Handlung einsetzt und zum Gegenstand von Reflexionen der Hauptfigur macht, ist sonst in keinem anderen Text der Popliteratur zu finden. Hierin markiert der Roman einen Höhepunkt der literarischen Konstruktion von Körperausscheidungen im popliterarischen Diskurs. Unter der Perspektive einer Körper(selbst)kontrolle wird zugleich in der vorgeführten Aufnahme und Abgabe von Sekreten nach den Möglichkeiten und Grenzen eines beherrschbaren und damit kontrollierbaren Körpers gefragt. Der geöffnete Körper wird auf zwei Ebenen kontextualisiert: einerseits

23 Vgl. zur Ästhetik der Oberfläche: Seiler: "Das einfache wahre Abschreiben der Welt « oder den von Grabienski u.a. herausgegebene Sammelband zur deutschsprachigen Popliteratur Poetik der Oberfläche. 
im Rahmen einer lustvollen, experimentellen Entgrenzung, andererseits in dem einer gewaltsamen und unkontrollierten Öffnung.

\section{Lustvolle Entgrenzung oder die hyperstilisierte Überschreitung der Norm}

Die Ich-Erzählerin pflegt »einen sehr engen Kontakt « ${ }^{24}$ zu ihren Körperausscheidungen. Helens Körpersekrete befinden sich in einem ständigen Kreislauf des Ausscheidens und Wiederaufnehmens. Sie verwendet ihr Smegma »wie andere ihre Parfümflakons $\aleph^{25}$, tauscht mit ihrer Freundin benutzte Tampons als Zeichen ihrer »Blutsschwesternschaft $«^{26}$ und führt seit Jahren ein $»$ Muschihygieneselbstexperiment ${ }^{27}$ durch, indem sie sich auf beschmutzte öffentliche Toiletten setzt, um hierbei gezielt möglichst allerlei Bakterien, Bazillen und fremde Körpersäfte aufzunehmen. Im gleichen Maße verteilt sie ihre Sekrete an öffentlichen Orten und schreckt auch nicht davor zurück, ihre eigenen Ausscheidungen wieder aufzunehmen, ganz im Sinne einer »Körperausscheidungsrecyclerin ${ }^{28}$.

Ich kann tatsächlich von nichts an meinem Körper die Finger lassen. Ich finde für alles eine Verwendung. Wenn ich merke, dass langsam ein kleiner Popel in der Nase hart wird, muss ich den rausholen. Als ich noch kleiner war, habe ich das sogar in der Klasse gemacht. Ich kann auch heute noch nichts Schlimmes daran finden, wenn jemand seine Popel isst. ${ }^{29}$

Die hier thematisierte und kontrollierte Überschreitung der Körpergrenze durch Aufnahme und Abgabe von eigenen und fremden Körpersekreten steht im direkten Kontrast zu einem Körperkonzept, das die Verschließung des Körpers fordert und innerhalb der Erzählung durch die Mutterfigur repräsentiert wird. Die Mutter Helens wird als Vertreterin eines Körperideals gezeichnet, das Körperausscheidungen als Verletzung eines ästhetischen und hygienischen Diktates sofort zu beseitigen und einzugrenzen sucht.

Meine Mutter hat auf meine Muschihygiene immer großen Wert gelegt [...]. Aus Muschiwaschen wird bei uns zu Hause eine riesenernste Wissenschaft gemacht. Es ist angeblich sehr schwierig eine Muschi wirklich sauberzuhalten. ${ }^{30}$

Mama findet, das Wichtigste für eine Frau, die ins Krankenhaus kommt, ist, saubere Unterwäsche anzuhaben [...]. Mama stellt sich, glaube ich, vor, dass alle im Krankenhaus dann rumerzählen, was Frau Memel für eine dreckige Schlampe ist. Außen hui, untenrum pfui. ${ }^{31}$ 
Ebenso, wie die Vagina der Tochter einem zu kontrollierenden und anzuleitenden Reinigungszwang unterworfen ist, werden auch die Ausscheidungen des Afters seitens der Mutter in Form von Hypertypen negiert: »Als ich ein kleines Mädchen war, hat sie mir oft gesagt, sie gehe nie groß auf Toilette. Sie müsse auch nie furzen. Sie behalte alles innen, bis es sich auflöse. $\aleph^{32}$ Helens offensiver Umgang mit den Sekreten ihres eigenen, aber auch jener fremden Körper, kann nach Julia Reichenpfader als Form des Protestes gegen die Ideale ihrer Mutter gelesen werden. ${ }^{33}$ Hierbei wird der Körper Helens zum Subjekt ihrer eigenen Lust und zur Auflehnung gegen jene "Hygienefanatiker " ${ }^{34}$ die eine vollständige Verschließung des Körpers fordern. Die kontrollierte und hyperstilisierte Überschreitung der Körpergrenze wird innerhalb der Erzählung aus Perspektive der IchErzählerin als ein experimentelles und lustvolles Spiel entgegen der Norm der Elterngeneration konstruiert. Diese kontrollierte Überschreitung der Körpergrenze in der Figur der Helen Memel wird durch Roche positiv besetzt, denn die Autorin arbeitet im Bereich der verschiedensten Sekrete ohne den Einsatz von Verwerfungsprädikaten, ${ }^{35}$ die eine eindeutig negative Lesart der vorgeführten Körperausscheidungen und der Aufnahme der selbigen rechtfertigen würden. Darin verzichtet die Verfasserin auf die Kontextualisierung der Sekrete im Bereich des Ekels und/oder des Hässlichen. Eine derartige Kontextualisierung, wie sie beispielsweise von zahlreichen Kritikern vorgenommen wurde, findet ausschließlich im Rahmen eines Rezeptionsprozesses statt, der den verschlossenen Leib als Ideal setzt. In dem Verzicht auf den Einsatz von Verwerfungsprädikaten im Kontext der Körpersekrete folgt Charlotte Roche zahlreichen Autoren der 1990er Jahre, wie z.B. Christian Kracht oder Alexa Henning von Lange. ${ }^{36}$

\section{Der eingeschriebene Normierungszwang}

Eine vollständige Distanzierung von den durch die Mutterfigur repräsentierten Körperidealen gelingt Helen jedoch nicht:

Dass ich in Muschisachen so gesund und in Arschsachen normalerweise so verkrampft bin, liegt daran, dass meine Mutter mir ein Riesenkackaproblem angezüchtet hat. [...] Wegen solcher Erzählungen schäme ich mich total, wenn jemand mich auf Klo hören oder riechen kann. Auf einer öffentlichen Toilette, auch wenn ich nur pinkele [...] werde ich

33 Vgl. Reichenpfader: Verletzte Hüllen, fehlende Häute, S. 343.

34 Roche: Feuchtgebiete, S. 19.

35 Vgl. zum Verständnis der Verwerfungsprädikate: Meinen: Eine Ästhetik des Ekels, S. 120.

36 Vgl. ebd., S. 120-124. 
um jeden Preis verhindern, dass die Frau in der Kabine neben mir das Gesicht zu dem Geräusch zu sehen bekommt. Genauso benehme ich mich auch bei meinem Kackageruch. Wenn reges Kommen und Gehen in der Kabine neben mir herrscht und ich rumgestunken habe, bleibe ich so lange ruhig in meiner Kabine, bis kein Zeuge mehr da ist. Dann erst traue ich mich raus. ${ }^{37}$

Diese Passage zeigt deutlich, dass, obwohl die Erzählfigur zur Reflexion dieser hyperstilisierten Normierung der Fäkalien durch die Mutterfigur fähig ist, sie dennoch nicht in der Lage ist, sich dieser zu widersetzen. Hierin zeichnet Roche in der Figur Helen Memel einen in den Körper eingeschriebenen Normierungszwang nach. Die Schamhaftigkeit, mit der Helen ihre Fäkalien belegt, wird auch im Bereich der Sexualität sichtbar. Obwohl der Po bei Helen zum Sex dazu gehört, ist sie, im Gegensatz zu ihrer sonstigen Freizügigkeit, zum Austausch der Fäkalien nur bereit, wenn sie »schon ein paar Mal guten Sex mit ihm hatte. Das ist ein großer Liebesbeweis, den ich dann erbringe. Analsex, ohne dass ich mir den Arsch vorher ausgespült habe. Da muss das Vertrauen groß sein. « ${ }^{38}$

Minutiös beschreibt die Autorin daher die Reinigung des Afters, die Helen derart perfektioniert hat, dass sie "präpariert « ist »für sauberen Posex, wie eine Gummipuppe ${ }^{39}$ In dieser Beschreibung wird die Perfidität und Unnatürlichkeit dieser Körperperfektion aus Sicht der Erzählfigur konstruiert. Hierzu zählt auch, dass Helens Po »diesem modernen Rasurzwang unterworfen $\aleph^{40}$ ist. Obwohl Helen sich »innerlich sehr gegen das Rasieren $\aleph^{41}$ und damit gegen das ästhetische Konzept einer glatten Körperoberfläche sträubt, beugt sie sich dennoch diesen ästhetischen Anforderungen. Das Körperbild einer glatten und damit haarlosen weiblichen Körperoberfläche wird im Text als die Normierung des weiblichen Körpers durch den Mann vorgestellt: »Ich finde, wenn Männer rasierte Frauen wollen, sollen sie auch das Rasieren übernehmen. Und nicht den Frauen die ganze Arbeit aufhalsen. Frauen wäre es doch ohne Männer egal, wie sie behaart sind. ${ }^{42}$

Die Fäkalien und der After markieren im Text den Punkt größter Intimität und sind somit zugleich höchster (Selbst)Kontrolle und Disziplin unterworfen. Der Umgang mit den Fäkalien wird durch die Hauptfigur als die widerwillige Anerkennung eines in den Körper eingeschriebenen Normierungszwanges konstruiert. Die Anerkennung dieses Körperideals führt

38 Ebd., S. 91.

39 Ebd.

40 Ebd., S. 10.

41 Ebd.

42 Ebd., S. 67. 
jedoch zur gewaltsamen Öffnung des Körpers, denn bereits zu Beginn der Handlung steht der geöffnete und nicht mehr kontrollierbare Körper im Fokus der Handlung: »Alles das Ladyshaven schuld. Feel like Venus. Be a goddess! «33

\section{Die gewaltsame Öffnung}

Der After als scheinbar kontrollierbare Grenze zwischen Innen und Außen wird durch die Verletzung Helens und die sich anschließende Notoperation durchschritten, die Grenze wird aufgehoben. Hier findet durch die institutionellen Vertreter des Krankenhauses ein Eingriff in Helens Körper statt, der durch den medizinischen Diskurs legitimiert, aus der Perspektive der Figur jedoch als eine übergriffige Handlung verstanden wird.

Der nächste Proktologe, der reinkommt, sagt kurz: »Guten Tag, Professor Dr. Notz mein Name. «Und rammt mir dann was ins Arschloch. Der Schmerz bohrt sich die Wirbelsäule hoch bis zur Stirn. [...] Nach ein paar Schrecksekunden habe ich ein platzendes, nasses Gefühl und schreie: »Aua, vorwarnen bitte. Was war das, verdammt? « Und er: »Mein Daumen. [...] "Was für eine Art sich vorzustellen. ${ }^{44}$

Im Anschluss an die Operation steht Helens Körper nun offen und nichts deutet mehr auf einen geschlossenen Schließmuskel hin:

Mehr Loch als Arsch. ${ }^{45}$

Ich befürchte, dass ich auf jeden Fall schon mal luftinkontinent am Arsch bin. Es kommt einfach andauernd ohne jede Vorwarnung warme Luft aus dem Darm. Einen Furz kann man das beim besten Willen nicht nennen. Ich stehe an der Stelle ja einfach offen. [...] Es dampft einfach alles raus. ${ }^{46}$

Die von Helen erfahrene Unkontrollierbarkeit ihres Körpers wird im Roman vorrangig durch die Personifikation einzelner Teile des Körpers markiert, den Roche hierin als »(eigen)mächtig « ${ }^{47}$ konzipiert: »Der Körper und ich machen ein inneres Mpft, und dabei kommt ein Schwall von etwas unten raus. Warm. Könnte alles sein. Aus jeder Öffnung. $\aleph^{48}$

Die Hauptfigur unternimmt zahlreiche Versuche, die Kontrolle über ihren Körper wiederzuerlangen. Hierzu zählt die Bitte an den Krankenpfleger Robin, ihre Verletzung fotografisch festzuhalten, damit sie sich ein eigenes Bild ihres Afters machen kann, und die Versuche, ihre Wunde selbst zu versorgen. 
Die Fäkalien, die in den Rückblenden als jene Körperausscheidungen konzeptualisiert wurden, die durch die Übernahme eines durch die Nebenfiguren repräsentierten Normierungszwanges höchster Überwachung unterworfen sind, stehen im Handlungsverlauf stellvertretend für den Verlust der Körperkontrolle.

\section{Heinz Strunk: Fleckenteufel}

Vor Aufregung und Hektik und Angst habe ich heute noch gar nicht gekackt. Und gestern auch nicht. Das rächt sich, ausgerechnet jetzt! Ich könnte die Toilette des Gemeindehauses benutzen, aber Kacken ist etwas Schmutziges, das man privat für sich machen muss. Ich lasse erst mal eine Ladung Entlastungspupse kommen. Pppppffff. ${ }^{49}$

Mit der Erzählung des versäumten Toilettenbesuches des pubertierenden Ich-Erzählers Thorsten Bruhns beginnt der Roman Fleckenteufel von Heinz Strunk. Strunk präsentiert in Fleckenteufel, wie auch in seinen früheren Erzählungen (Fleisch ist mein Gemüse, 2004; Die Zunge Europas, 2008), eine tragisch-komische Geschichte scheinbarer Unzulänglichkeiten. Thorsten nimmt im Sommer 1977 an einer Familienfreizeit der evangelischen Gemeinde in Scharbeutz an der Ostsee teil. Er ist 16, träumt von Sex mit gleichaltrigen Jungen und Mädchen, liest Fünf-Freunde-Bücher und leidet unter erheblichen »Kackhemmung[en] « ${ }^{50}$ Die Erzählung wechselt zwischen den strukturierten Abläufen der christlichen Jugendfreizeit und den Beschreibungen eines als unzulänglich verstandenen jugendlichen Körpers. Der Leser erfährt von Thorstens Teilnahme an trostlosen Gruppenspielen und seiner akribischen "Rosettenpflege«, von miserablen Essensrationen und steifen Andachten, von Flatulenzen und Verstopfung. In Fleckenteufel sind die Fäkalien in einer regelrechten Typologie der Darmbewegungen und -ausscheidungen das Leitmotiv. Die Ausscheidungen des Darms werden ebenso detail- wie facettenreich unter Einsatz onomatopoetischer Mittel beschrieben und thematisiert, bis hin zu der Frage, warum der Mensch den eigenen Furz gerne riecht, nicht jedoch das eigene Erbrochene. ${ }^{51}$

In Fleckenteufel wird die Tragik und Komik der Diesseitsbewältigung des jugendlichen Helden durch dessen Versuche, die Kontrolle über die Regungen seines Körpers zu erlangen, markiert. Hormonen und Enzymen scheinbar hilflos ausgeliefert, durchlebt Thorsten Momente größter Peinlichkeit und Scham, aber auch des Triumphes, denn alles scheint »nur eine

50 Ebd., S. 64.

51 Vgl. ebd., S. 7. 
Frage der Kontrolle. Ein gut funktionierender Schließmuskel ist ebenso wichtig wie eine Lunge, die nicht dauernd in sich zusammenfällt «. ${ }^{52}$

\section{Von der Trockenlegung feuchter Gebiete}

Die Analogien des Romans Fleckenteufel zum Debüt Charlotte Roches in Covergestaltung, Schrifttyp und Titel sind offensichtlich. Das publizistische Feuilleton beschrieb den Roman Strunks als die männliche Antwort auf Roches Feuchtgebiete. In der »Welt « hieß es, Strunk stochere »in den Feuchtgebieten der Jungs « ${ }^{53}$ Silvia Kusidio schreibt in ihrer Rezension "auf die Feuchtgebiete folge der Fleckenteufel $\aleph^{54}$ und in der »taz « urteilt Julian Weber, Strunk ergänze mit seinem Roman Charlotte Roches Feuchtgebiete. ${ }^{55}$ Beide Erzählungen thematisieren im Motiv der Körperausscheidungen die Möglichkeiten, Kontrolle über den eigenen Körper zu erlangen. Doch während die Heldin der Feuchtgebiete ein im Text durch die Nebenfiguren präsentiertes Körperideal demontiert und sich damit dem Ideal eines verschlossenen Körperkonzeptes immer wieder entzieht oder dieses nur widerwillig anerkennt, kämpft die Figur Strunks um die ständige Aufrechterhaltung einer Körpernorm, die die Verschließung des Körpers fordert. Thorsten wird als Figur inszeniert, die, anders als die Protagonistin in Feuchtgebiete, das Ideal eines verschlossenen Körpers internalisiert hat.

\section{"Ist das alles peinlich ${ }^{56}$}

Thorsten schämt "sich zu Boden $«^{57}$ seit er denken kann. Besonders die unkontrollierten Regungen seines Leibes werden im Roman mit Scham und Peinlichkeit besetzt. Seine Flatulenzen sind »Wolken des Verderbens « ${ }^{58}$ und »Kacken ist etwas Schmutziges $«{ }^{59}$ Durch die Verwendung dieser Verwerfungsprädikate verortet Strunk die Fäkalien - aus Perspektive der Erzählfigur - im Diskurs des Ekels und des Schmutzes. Diese Konstruktion impliziert die Anerkennung eines Körperideals, das die Ausscheidungen des Körpers mit zahlreichen Ver- und Geboten belegt. Aus Figurenpers-

Ebd.

53 Cassier/Schmiechen: Strunk stochert in den Feuchtgebieten der Jungs.

54 Kusidio: Auf die »Feuchtgebiete" folgt der »Fleckenteufel «.

55 Vgl. Weber: Magna Charta der Darmwindungen.

56 Strunk: Fleckenteufel, S. 7.

57 Ebd.

58 Ebd., S. 204.

59 Ebd., S. 6. 
pektive ermöglicht das Fehlen der öffentlichen Kontrolle im Privaten, die Ausscheidung von Fäkalien als Genuss zu erleben. Hierzu finden sich im Text zahlreiche Belege: »Kacken ist etwas «, so die Bewertung der Erzählfigur, »was man privat für sich machen muss«, fürs "Scheißen« auf dem Busklo »kommt man ins Gefängnis « und Flatulenzen im öffentlichen Raum werden von der Frau des Küsters mit einem »Kleine-Leute-Hassgesicht « ${ }^{60}$ unmittelbar bestraft. »Allein zu Hause « jedoch, so Thorsten, »kann man den Arschdruck auskosten und genießen. Arschdruck ist geiler als Kiffen. « ${ }^{61}$ Diese Textauszüge dokumentieren zweierlei: Einerseits die Lust des Heranwachsenden an den Regungen des eigenen Körpers jenseits bürgerlicher Tabus, andererseits jedoch die Anerkennung gesellschaftlicher Normen und Konventionen, die das Ausscheiden von Fäkalien in vielfacher Hinsicht reglementieren.

Thorstens Bestreben im Bereich der Körperausscheidungen die Norm eines kontrollierten >Ansich-Haltens` zu erfüllen, ist jedoch insgesamt von wenig Erfolg gekrönt, wie ein Blick in die bereits erwähnte Eingangsszene zeigt: Nachdem Thorsten vor Aufregung vergessen hat, seinen Darm vor der Abfahrt in die Jugendfreizeit zu entleeren, rächt sich dieser in Form eines Sturzdurchfalles im Garten des Pfarrhauses. So etwas Peinliches ist »ihm im Leben noch nicht passiert «. ${ }^{62}$ Sein Po ist nun wund und so wird bereits die Hinreise im vollbesetzten Bus zu einer schmerzhaften Erfahrung. Der unkontrollierbare und geöffnete Körper steht somit am Beginn der Erzählhandlung im Fokus. Auf die Überschreitung der Norm reagiert Thorstens Körper nun mit Verschließung. Im weiteren Verlauf wird er von starken Verstopfungen gepeinigt, die täglichen $»$ Kacksitzungen ${ }{ }^{63}$ sind ergebnislos, er wird »dicker und dicker « ${ }^{64}$ und befürchtet irgendwann $»$ Zu platzen $«{ }^{65}$ Die Fäkalien sind im Roman Ausdruck mangelnder Selbstkontrolle, indem sie sich der Beherrschbarkeit durch die Erzählfigur entziehen. Der eigene Körper wird dadurch als demütigend erfahren. Der Konnex von Fäkalien und Demütigung spiegelt sich auch im aggressiven Kommunikationsverhalten der Jugendlichen untereinander wider, wenn einer der Mitreisenden Thorsten mit der kontextfreien Verbalgeste beschimpft: »Hast du dir eigentlich schon mal in den Arsch gekackt? ${ }^{66}$

\footnotetext{
60 Ebd., S. 9.

61 Ebd., S. 11.

62 Ebd., S. 8.

63 Ebd., S. 144.

64 Ebd.

65 Ebd.

66 Ebd., S. 21.
} 
Im gleichen Maße, wie sich seine Verdauung seiner Selbstkontrolle entzieht, entziehen sich auch andere Lebensbereiche seiner Einflussnahme. Er scheitert ebenso beim Versuch, Teil der coolen Jungsclique zu werden wie von den Mädchen wahrgenommen zu werden. Selbst nach der gemeinsamen »Druckbetankung « mit Apfelkorn geht Thorsten bei der sich anbahnenden Knutscherei leer aus und wird von seinen Zeltkollegen im Freien zurückgelassen, wo er seinen Rausch ausschläft.

\section{"Ein Leben ohne Magen-Darm-Trakt, mit schweinchenrosa Rosette und ewig guter} Laune, das wär's. « ${ }^{67}$

Gerne würde Thorsten irgendwo dazugehören oder wäre auch gerne wie »irgendwer, damit er sich nicht so alleine auf der Welt fühle «. ${ }^{68}$ Der Wahrnehmung des vollständigen Kontrollverlustes und der Unmöglichkeit der Einflussnahme setzt Thorsten zwei Tätigkeiten entgegen: »Wichsen plus penibelste Analhygiene ${ }^{69}{ }^{69}$ Thorsten ergeht sich in hetero- und homosexuellen Fantasien gleichermaßen intensiv wie in der Vision eines zeitlos schönen Afters. Es ist sein erklärtes Ziel, bis ins hohe Alter seine »Rosette in einem absoluten Topzustand ${ }^{70}$ zu erhalten. In der akribischen Behandlung seines Afters mit Nivea-Creme glaubt er, dieses Ziel erreichen zu können:

Nach meinem Tod liege ich als eine unter unzähligen Leichen aufgebahrt in der Totenwaschanlage. Ich bin wie alle anderen total verrottet, mit Grieben, Flechten, Warzen und Geschwüren zugewachsen, grisseliges Haar überall und nirgends [...] Die Leichenwäscher stören sich nicht daran, sie sind einiges gewöhnt. [...] Dienst nach Vorschrift, alles wie immer, doch dann: Als sie meine Arschbacken auseinanderziehen, um mein Loch zu waschen, schimmert ihnen eine jungfräuliche Rosette entgegen, der Lohn jahrzehntelanger Reinigungsanstrengungen. So soll und wird es eines Tages kommen. ${ }^{71}$

Beide von der Erzählfigur präsentierten Lösungskonzepte sind Ausdruck der Notwendigkeit, der von Thorsten erfahrenen Hilflosigkeit etwas Beherrschbares entgegenzusetzen. Die Fokussierung der Erzählfigur auf eine Rosetten-Ästhetik stellt eine Parodie auf das Konzept eines ästhetischen, alle Körperbereiche durchdringenden Körperideals dar.

Die Erzählung Strunks endet mit dem Tod von Elvis Presley. Entgegen der offiziellen Version ist der Musiker in der Fantasie des Erzählers an 
Verstopfung gestorben: »mit dem Bauch aufgebläht von Tabletten, Burgern und vertrockneter alter Scheiße. Der King an Verstopfung gestorben [...]. « ${ }^{72}$

\section{Fazit}

Roche und Strunk folgen in ihren Darstellungen der allgemeinen Tendenz popliterarischer Texte, den eigenen Körper, dessen Öffnungen und Ausscheidungen zentral zu inszenieren und sich hierin dem »Gebot der empfindsamen Entkörperlichung des Begehrens ${ }^{73}{ }^{73}$ zu widersetzen. Die Texte schreiben in der hyperstilisierten Konzentration auf die verschiedenen Ausscheidungen des Körpers von der Wirkmacht eines Körperdiskurses, der einzig die kontrollierte, durch gesellschaftliche Normen konventionalisierte Überschreitung der Körpergrenzen legitimiert. Die Texte präsentieren in der bewussten Überschreitung der Körpergrenzen und der Erkundung des Körperinneren das Bild von einem lustvollen Spiel mit dem eigenen Körper und dessen Funktionen. In diesen Darstellungen zeigt sich ein Widerstand gegen Disziplinierung und Normierung. Die konventionalisierten Grenzübertritte werden in den Handlungszusammenhängen unterlaufen und aus Figurenperspektive positiv erlebt und bewertet. Wie stark der Verschließungsdiskurs und seine Normierungen dennoch auf die Figuren einwirken, zeigt sich darin, dass der nicht-konventionelle Grenzübertritt nur in einzelnen Bereichen stattfindet und der individuellen Kontrolle unterliegt. Bemerkenswerterweise arbeiten sowohl Roche als auch Strunk mit adoleszenten Figuren, denen eine Grenzüberschreitung im literarischen Rezeptionsprozess in verschiedensten Bereichen am ehesten zugestanden wird. Das Konzept einer lustvollen selbstbestimmten Entfernung vom Ideal des verschlossenen Köpers erscheint hier nicht als grundsätzlicher Anspruch, sondern als Stufe des Heranwachsens.

Wie stark jedoch die Wirkmacht des Diskurses ist, zeigt sich dann, wenn den Figuren die Kontrolle über den eigenen Körper versagt: Dann dominieren Scham und Peinlichkeit. Dies geschieht in den Erzählungen vorrangig im Bereich der Fäkalien. In beiden Romanen fürchten sich die Protagonisten vor dem Versagen ihres Schließmuskels. Obwohl die Figuren zur kritischen Reflexion der konventionalisierten Normierung des Ausscheidens fähig sind, gelingt ihnen eine vollständige Distanzierung von dem Ideal eines verschlossenen Körpers in der Figur der Fäkalien nicht. Darin offenbart sich

73 Degler: Selbstbezüglichkeit, S. 87. 
ein Widerspruch, denn ausgerechnet in der Anerkennung der Norm wendet sich die Darstellung ins Pathologische. Helens Selbstverletzung wird durch die Institution Krankenhaus so weit vorangetrieben, dass sie Gefahr läuft, tatsächlich "anal inkontinent ${ }^{74} \mathrm{zu}$ werden, und Thorsten reagiert auf das Bewusstsein seiner Normverletzung mit Verstopfung. In der Anerkennung der verschlossenen Körpernorm kommt es zum vollständigen Kontrollverlust. Hierin zeigt sich der subversive Charakter der Darstellungen.

\section{Literaturverzeichnis}

Bachtin, Michail: Rabelais und seine Welt. Volkskultur als Gegenkultur. Hg. Renate Lachmann. Frankfurt/M.: Suhrkamp 1995.

Bartels, Klaus: Trockenlegung von Feuchtgebieten. Christian Krachts Dandy-Trilogie. In: Poetik der Oberfläche. Die deutschsprachige Popliteratur der 1990er Jahre. Hgg. Olaf Grabienski, Till Huber, Jan-Noel Thon. Berlin, Boston: De Gruyter 2001, S. 207-225.

Benthin, Claudia: Haut. Literaturgeschichte - Körperbilder - Grenzdiskurse. Reinbek/H.: Rowohlt 2001.

Bessing, Joachim; Kracht, Christian u.a.: Tristesse Royale. 3. Aufl. Berlin: List 2009.

Cassier, Philipp; Schmiechen, Frank: Strunk stochert in den Feuchtgebieten der Jungs. »Welt online« (5.2.2009). <https://www.welt.de/kultur/article3154893/Strunk-stochert-inden-Feuchtgebieten-der-Jungs.html $>$ (Zugriff: 18.3.2018).

Degler, Frank: Sekrete Kommunikation. Das Motiv der Körperflüssigkeiten in der Neuen Deutschen Popliteratur. In: Epochen/Krankheiten. Konstellationen von Literatur und Pathologie. Hgg. Frank Degler, Christian Kohlroß. St. Ingbert: Röhrig Universitätserlag 2006, S. 265-287.

Degler, Frank; Paulokat, Uta: Neue Deutsche Popliteratur. Paderborn: Wilhelm Fink Verlag 2008.

Degler, Frank: Selbstbezüglichkeit. Sex und Gender in »Relax« und »Soloalbum«. In: Pop und Männlichkeit. Zwei Phänomene in prekärer Wechselwirkung? Hg. Katja Kauer. Berlin: Fink \& Timme 2009, S. 71-88.

Douglas, Mary: Reinheit und Gefährdung. Eine Studie zu Vorstellungen von Verunreinigung und Tabu. Frankfurt/M.: Suhrkamp 1988.

Elias, Norbert: Über den Prozess der Zivilisation. Soziogenetische und psychogenetische Untersuchungen. Erster Band. Wandlungen des Verhaltens in weltlichen Oberschichten des Abendlandes. Frankfurt/M.: Suhrkamp 1997.

Grabienski, Olaf; Huber, Till; Thon, Jan-Noel: Poetik der Oberfläche. Die deutschsprachige Popliteratur der 1990er Jahre. Berlin, Boston: De Gruyter 2001.

Heimerl, Theresia: Der Skandal des Körpers. Woran Feuchtgebiete die Theologie erinnern sollte. »Herder Korrespondenz. Monatsheft für Gesellschaft und Religion« 11 (2008), S. 562-567.

Henning von Lange, Alexa: Relax. 10. Auflage. Reinbek/H.: Rowohlt 2008. 
Hörisch, Jochen: Ende der Vorstellung. Die Poesie der Medien. Frankfurt/M.: Suhrkamp Taschenbuch Verlag 1999.

Kracht, Christian: Faserland. 9. Auflage. Köln, Frankfurt/M.: Kiepenheuer \& Witsch 2018. Kracht, Christian: 1979. 5. Auflage. Köln: Kiepenheuer \& Witsch 2005.

Kusidio, Silvia: Auf die »Feuchtgebiete «folgt der »Fleckenteufel«. »Mitteldeutsche Zeitung « (12.2.2009). <https://www.mz-web.de/kultur/literatur-auf-die-feuchtgebiete-folgtder-fleckenteufel-7954546> (Zugriff: 12.9.2017).

Löw, Marie: Der Körperraum als soziale Konstruktion. In: Geschlechter-Räume: Konstruktion von "gender « in Geschichte, Literatur und Alltag. Hg. Margarethe Hubrath. Köln, Weimar, Wien: Böhlau Verlag 2001, S. 211-222.

Meinen, Iris: Eine Ästhetik des Ekels. Körperflüssigkeiten und Popliteratur. In: Was wir lesen sollen. Kanon und literarische Wertung am Beginn des 21. Jahrhunderts. Hgg. Stefan Neuhaus, Uta Schaffers. Würzburg: Königshausen \& Neumann 2016, S. 113-124.

Meninnghaus, Winfried: Ekel. Theorie und Geschichte einer starken Empfindung. Frankfurt/M.: Suhrkamp 1999.

Piepgras, Ilka: Ein Autor sinniert über seine Gedanken. »Berliner Zeitung« (23.3.1995). $<$ http://www.berliner-zeitung.de/archiv/ein-zeitgeistbuch-von-christina-kracht-derautor-sinniert-ueber-seine-gednaken,10810590,8931056.html> (Zugriff: 13. 5.2018).

Reichenpfader, Julia: Verletzte Hüllen, fehlende Häute. Frauenkörper in der deutschen Gegenwartsliteratur. In: Hülle und Haut: Verpackung und Umschlag, Techniken des Verkleidens und Umschließens. Hg. Ute Seiderer, Michael Fisch. Berlin: RotbuchVerlag 2014, S. 333-352.

Roche, Charlotte: Feuchtgebiete. 29. Aufl. Köln: DuMont Buchverlag 2008.

Rytz, Juliane Rosemarie: „Die Sprache ist eine Haut «. Subjektivierung entlang versehrter Körpergrenzen in der Gegenwartsliteratur. 2009. <https://elib.suub.uni-bremen.de/ edocs/00103943-1.pdf> (Zugriff: 12. 9.2017).

Seeßlen, Georg: Bedeutis und Wixis. Was kann die Popliteratur, und was meint sie zu können. »literatur konkret« 26 (2001/ 02). <https://konkret-magazin.de/hefte/sonderhefte/ id-26/articles/bedeutis-und-wixis.html> (Zugriff: 21.10.2017).

Seiler, Sascha: »Das einfache wahre Abschreiben der Welt«. Pop-Diskurse in der deutschen Literatur nach 1960. Göttingen: Vandenhoeck \& Ruprecht 2006.

Stuckrad-Barre, Benjamin: livealbum. Köln: Kiepenheuer \& Witsch 1999.

Strunk, Heinz: Fleckenteufel. Reinbek: Rowohlt Taschenbuch Verlag 2010 (erstmals 2009).

Weber, Julian: Magna Charta der Darmwindungen. »taz« (27.1.2009). <http://www.taz. de/!5168919/> (Zugriff: 23.9.2017).

Wittrock, Claudia: Anders sein - echt sein. Zur Attraktivität des versehrten Körpers in der jungen deutschsprachigen Gegenwartsliteratur. Bremen: Universität Bremen, Institut für kulturwissenschaftliche Deutschlandstudien 2000. <http://www.deutschlandstudien.uni-bremen.de/wp-content/uploads/2011/05/heft151.pdf> (Zugriff: 23.9.2017). 